\title{
Data Sharing Between Ada and C/C++
}

\author{
Matt Mark \\ Lockheed Martin \\ 9211 Corporate Blvd. \\ Rockville, MD 20850 \\ 301-640-2861 \\ matt.mark@Imco.com
}

\begin{abstract}
The En Route Automation Modernization (ERAM) program is a real-time Air Traffic Control (ATC) program being developed by Lockheed Martin Corporation. The ERAM program has high availability requirements, mission critical applications, and stringent response time requirements. The estimated size of the ERAM program is $1,300 \mathrm{KSLOC}$ and includes primarily Ada, C, and $\mathrm{C}++$ code. Legacy code being reused in ERAM is both Ada and $\mathrm{C}$. This resulted in the need for several cross-language interfaces. Standard methods for passing binary data structures between the languages have been developed and will be discussed in this paper.
\end{abstract}

\section{Categories and Subject Descriptors}

D.2.7 [Distribution, Maintenance, and Enhancement]: Restructuring, reverse engineering, and reengineering Language Contructs and Features. D.3.3 [Language Constructs and Features]: Data types and structures.

\section{General Terms}

Performance, Languages

\section{Keywords}

Cross-language Interface

\section{INTRODUCTION}

Lockheed Martin ATC programs prior to ERAM have had a limited number of cases where Ada and $\mathrm{C}$ source code shared data structures. Ensuring the structures used in both languages are equivalent had been done manually. Code commentary was normally included next to the data structure definition in both languages indicating that if the structure was changed, the equivalent structure in the other language must also be changed. This manual maintenance strategy is cumbersome at best.

The ERAM program has many more cases of data sharing between languages than our previous programs and a more rigorous approach was needed. For years, Lockheed Martin ATC programs have had utilities that use the Ada Semantic Interface

Permission to make digital or hard copies of all or part of this work for personal or classroom use is granted without fee provided that copies are not made or distributed for profit or commercial advantage and that copies bear this notice and the full citation on the first page. To copy otherwise, or republish, to post on servers or to redistribute to lists, requires prior specific permission and/or a fee.

SIGAda'05, November 13-17, 2005, Atlanta, Georgia, USA.

Copyright 2005 ACM 1-59593-185-6/05/0011...\$5.00.
Specification (ASIS) to evaluate Ada data structures and put information about them into a code-independent format[1]. This format is commonly referred to as a dictionary entry for the data structure. The dictionary entry includes the name of the type, name of fields within the type and the type names of the fields, the bit offsets of each field, the range of each field, etc. The process of creating a dictionary entry is commonly referred to as "tooling".

Prior to ERAM, the tooling of data structures into dictionaries was mainly done for use by support (offline) software that did not have a direct interface to operational code. Two examples of this use are:

1. Utility programs that convert text to binary files used as input (adaptation) data files for operational code, and

2. Utility programs that convert binary data structures which are recorded for system analysis. Dictionary entries are used to interpret the binary data recorded and produce text.

For ERAM, the ability to create dictionary entries from $\mathrm{C}$ and $\mathrm{C}++$ types was developed.

The approach taken to sharing of data between languages is to write utilities that:

1. Create compilable code containing data structures that have been tooled (referred to as type generation).

2. Compare two tooled data structures to determine if they are binary compatible (referred to as type matching).

With these utilities in place, the following steps are followed to address cross-language sharing of data:

- Define the structure to be shared between languages in Ada, $\mathrm{C}$, or $\mathrm{C}++$.

- Tool the type to create a dictionary entry.

- Run the type generator program which will create data structures in both Ada and C, regardless of the language of the original type.

- Use the "opposite language" data structure where needed.

- Tool the "opposite language" data structure.

- At system build time, the type matching tool is used to ensure compatibility of the two types.

The steps and more details about the type generator and type matching utilities will be discussed further in this paper. Also, 
other applications of these utilities will also be discussed, including platform and/or compiler migration.

Alternatives to sharing data structures between languages include converting data to an ASCII format such as XML. XML-based schemes have the advantage of being compiler, language and machine architecture independent, but at some cost. The scheme of sharing data structures detailed in this paper was developed mainly for performance considerations; the structures being shared are included in Ada and C++ APIs that are expected to be called hundreds of times per second. This scheme is a relatively low-maintenance approach to achieve the necessary run-time performance goals.

\section{TYPE GENERATOR}

The type generator is only an aide to help in producing binary compatible types. The developer that maintains the code produced by the type generator has the option of changing the types. This might be done for readability (the type generator selects names which the maintainer may wish to change) or to combine types.

The type generator produces an Ada package and C header file for each type given as input. Two types being run through the type generator could, for example, share underlying data structures which should be defined once in source code used by both higher level types.

The Ada package name and the $\mathrm{C}$ header file name used in the generated source code files are parameters to the type generator tool.

\subsection{Limitations}

There are limitations to the types that can be produced by the type generator. In order to share structures between Ada and C, only structures that had common attributes between the two languages can be processed. Also, since dictionary entries are being used as input, additional limitations are imposed.

These limitations include:

- Ada constant and C \#define statements are not supported. Information about constants is not included in dictionary entries and these values are not included in generated source code.

- Ada variant records (with variant parts or array bound discriminant) are not supported.

- $\quad$ C unions are not supported.

- Only floating point numbers used in $\mathrm{C}$ are supported. This means only 4 byte floats with 7 digits of precision and 8 byte floats with 15 digits of precision are supported. Ada fixed types are not supported.

- Integer types that are a multiple of 8 bits in size are supported. In Ada, a type that has a range from 0 .. 10 can be defined to be 4 bits. Such a type cannot be defined in C. Such a type can be included in a record in both Ada and C, but a type that is simply an integer of 4 bits cannot be defined in both languages.
- Floating point numbers and strings must be on byte boundaries. In Ada, these fields can be specified to not be on byte boundaries via rep-spec. This cannot be done in C.

- Arrays whose element size is not a multiple of 8 bits (a whole number of bytes) are not supported. For example, in Ada an array of two bit elements can be defined. This cannot be done in $\mathrm{C}$.

- A special case is handled, an array of bits that is a multiple of 8 in size. In this case, the generated $\mathrm{C}$ structure contains an array of characters whose size matches the Ada bit array.

\subsection{Special Cases}

There are several cases where the generated types differ from the original. The types generated and the original types are binary compatible (i.e. all primitive fields in the original type exist in the generated type as the same bit locations). We decided to put as few restrictions on the original type as possible. Because of this, the type generator program needed to handle some special cases.

\subsubsection{Subtypes}

Dictionary entries do not contain information about subtypes. The generated code will contain types that are not subtypes. For example, given the following Ada type Rec_T:

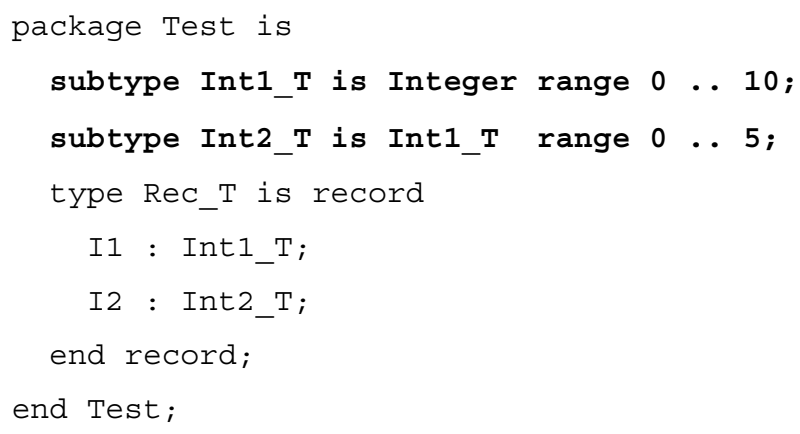

The type generator produces the following Ada package spec:

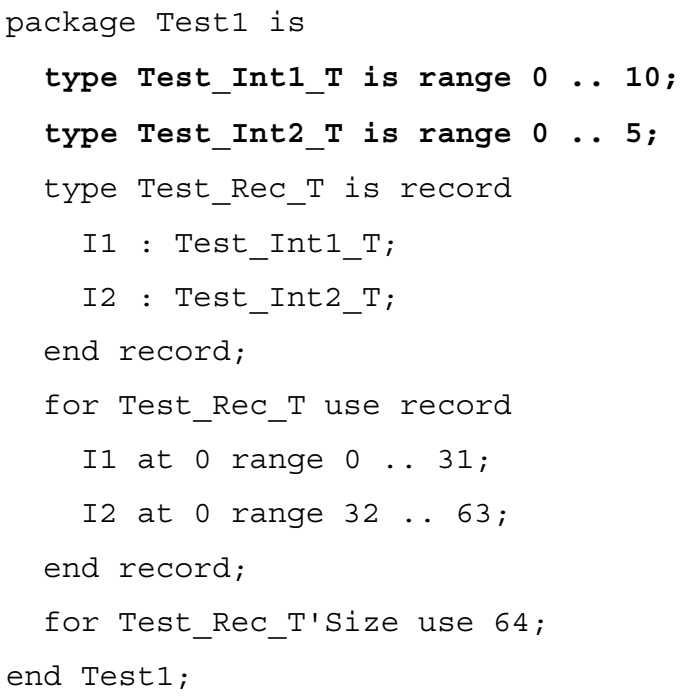


Generated type TEST INT1 T is not a subtype of Integer and TEST_INT2_T is not a subtype of TEST_INT1_T. This behavior would be difficult to change since it cannot be determined from the dictionary entry if a type is a subtype of another. However, the generated type specifies integer fields consuming the same bits as the original type.

\subsubsection{Case Sensitivity}

Ada is not case sensitive while $\mathrm{C}$ is. In $\mathrm{C}$, a field in a record can have the same name as the type, differing only by case. This cannot be done in Ada. We decided to handle this situation by appending "F" to the field name to ensure uniqueness. For example, given the following $\mathrm{C}$ type Rec_T:

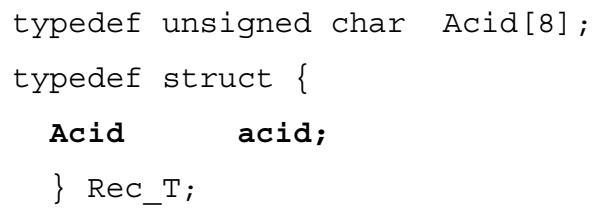

The type generator produces the following Ada package spec:

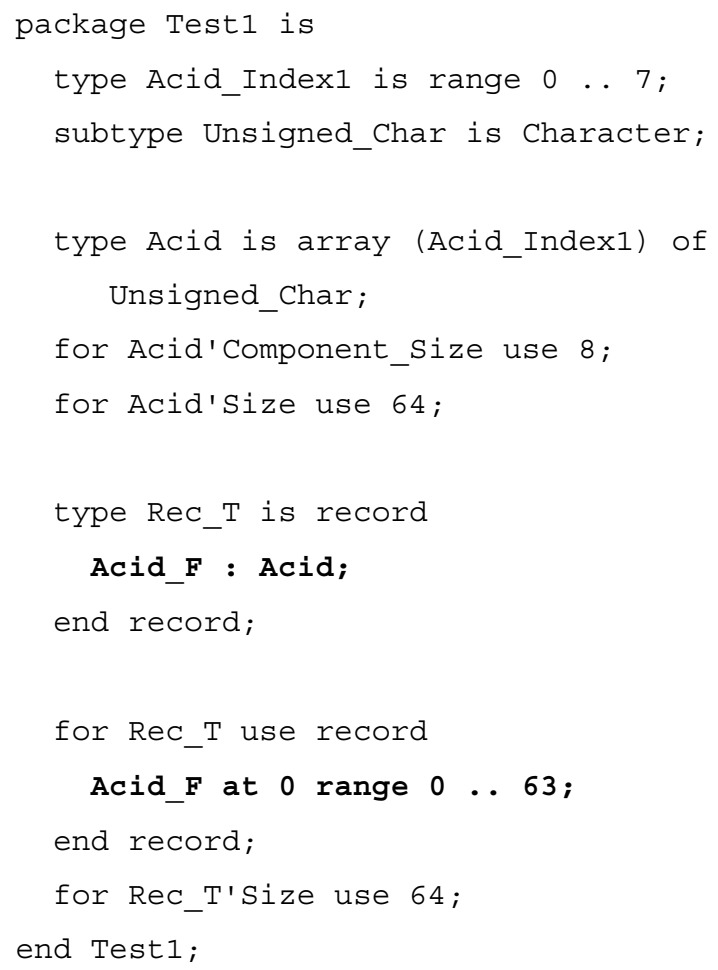

This behavior could easily be changed to reject types containing fields like these.

\subsubsection{Language Keywords}

Since Ada and $\mathrm{C}$ have different keywords, some type and field names in one language are not valid in the other. The type generator has some special code for keywords. For example, in the following type, field delay is a keyword in Ada.

typedef struct \{ signed int delay;
\} Rec_T ;

The type generator produces the following Ada package spec:

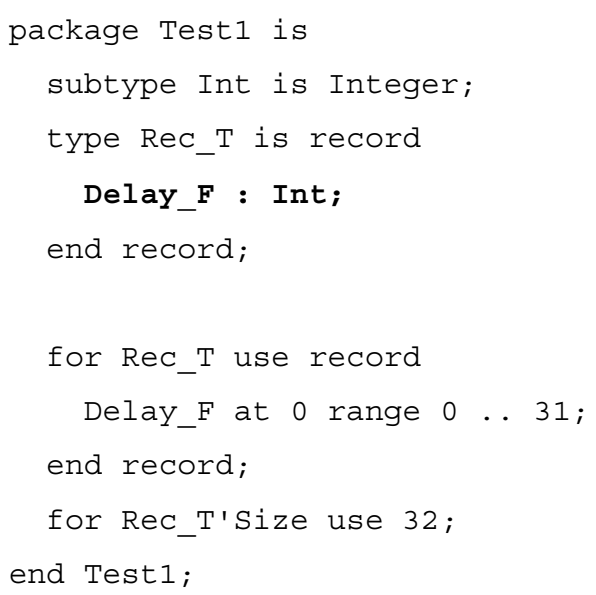

This behavior could easily be changed to reject types containing fields that are keywords in either language, but again, we decided to force different field names in this case.

\subsubsection{Enumeration Literals}

$\mathrm{C}$ does not allow an enumeration literal to be used more than once in a header file. The type generator has some special code to check for this case and ensure the all enumeration literals differ in the generated $\mathrm{C}$ header file. For example, in the following type, field DOWN is used in two enumeration types. This cannot be done in $\mathrm{C}$.

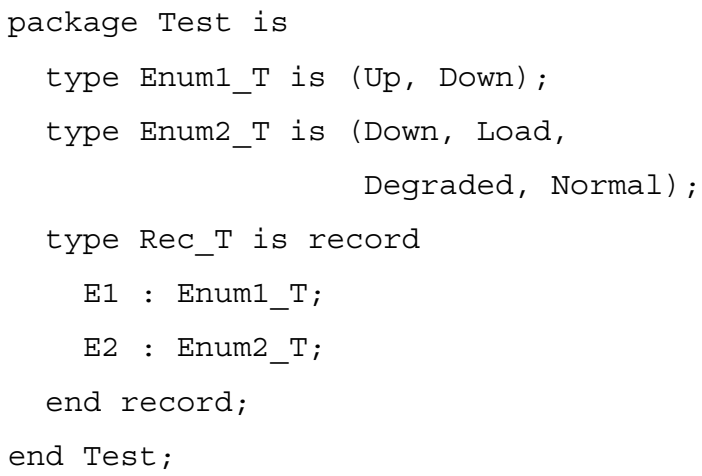

The type generator produces the following $\mathrm{C}$ header file:

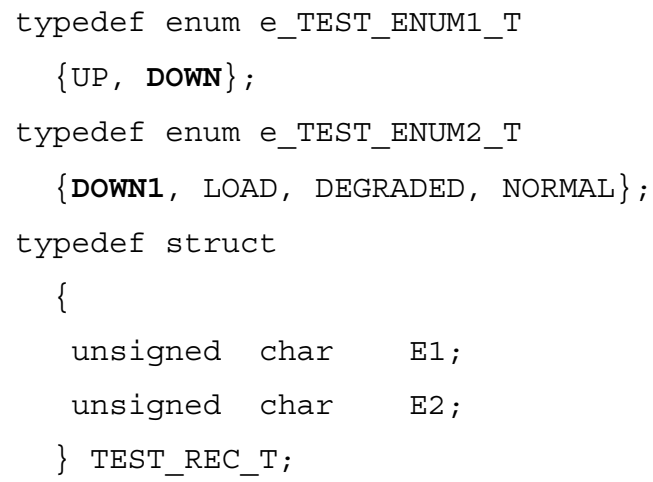


This behavior could easily be changed to reject types containing multiple enumerations with the same value. Again, we decided to force different enumeration literals.

\subsubsection{Enumerations in Records}

In $\mathrm{C}$ code created by the type generator, enumerations are not included as fields in records. Instead, an integer field of the appropriate size is put in its place. The reason for this is that in $\mathrm{C}$, enumerations cannot be specified to use fewer than eight bits while in Ada they can. For simplicity of the type generator program, we decided to always include integers in $\mathrm{C}$ structures where the original type had an enumeration. The previous example has been changed so that the enumerations in the original type are specified to be fewer than eight bits.

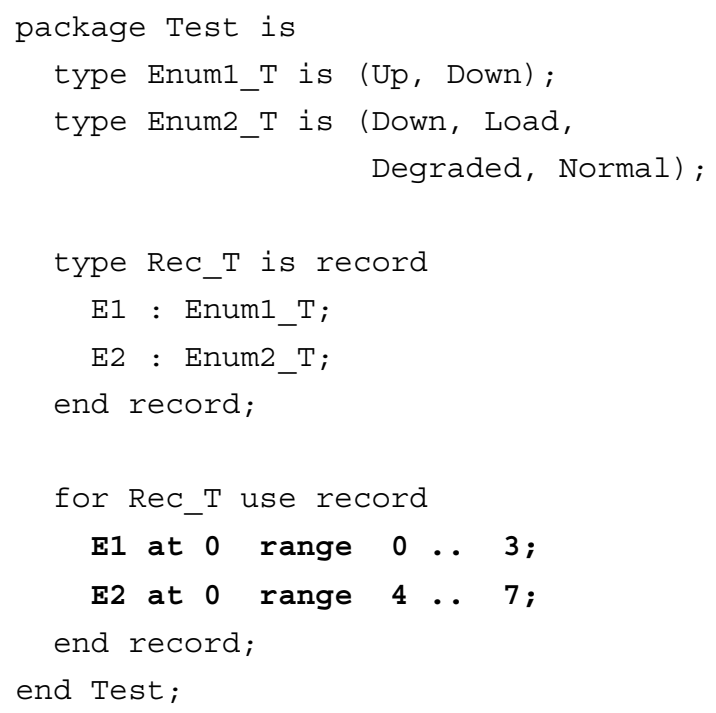

The type generator produces the following $\mathrm{C}$ header file:

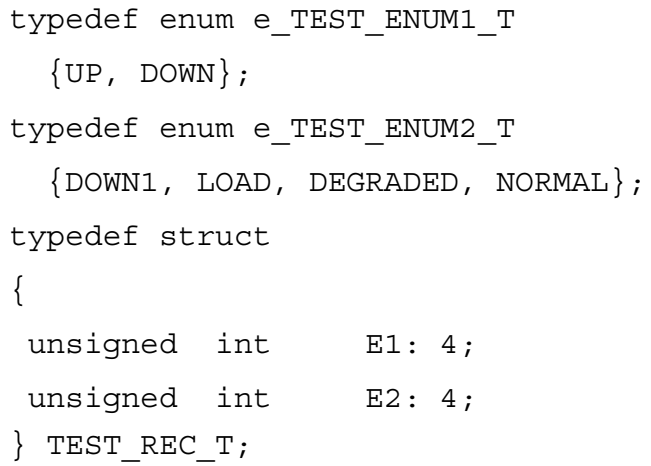

This behavior is needed to process enumerations contained in structures, where the enumeration consumes fewer than eight bits.

\subsubsection{Array Index Types}

For simplicity of the type generator program, array indices in Ada generated types are always defined as integers. This is done regardless of the original array index type (two discrete values, an integer type, an enumeration type, etc). For $\mathrm{C}$, the array is simply declared to have the correct number of elements.
Example 1: The below type is an array with a range of 1 .. Max_Elem (where Max_Elem is 3).

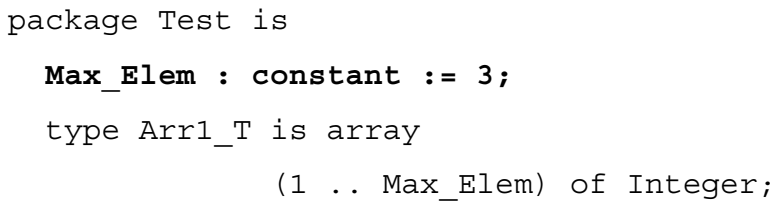

The type generator produces the following Ada package spec: package Test1 is

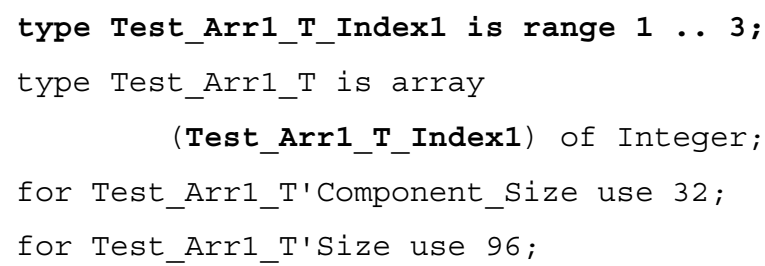

The following is the contents of the $\mathrm{C}$ header file produced:

typedef INTEGER TEST_ARRI_T[3] ;

Neither the generated Ada or C code contains the constant Max_Elem. Constants values are not in the dictionaries.

Example 2: The below type is an array with an enumeration, Enum_T, as the index.

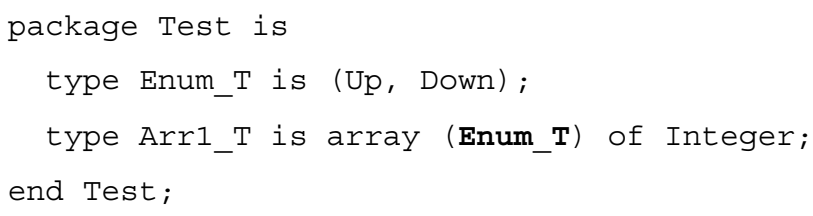

The type generator produces the following Ada package spec:

package Testl is

type Test_Arr1_T_Index1 is range $0 \ldots 1$;

type Test_Arr1_T is array

(Test Arr1 T Index1) of Integer;

for Test_Arr1_T'Component_Size use 32;

for Test_Arr1_T'Size use 64;

end Test1;

The following is the contents of the $\mathrm{C}$ header file produced:

typedef INTEGER TEST_ARR1_T[2];

Neither the generated Ada or $\mathrm{C}$ code contains the enumeration Enum_T. With a change to the type generator, it would be 
possible to use Enum_T as the array index in the generated Ada code, but this has not been done.

Example 3: The below type is an array with an integer type, Int_ $T$, as the index.

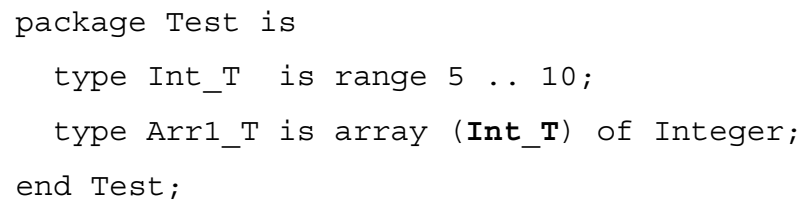

The type generator produces the following Ada package spec:

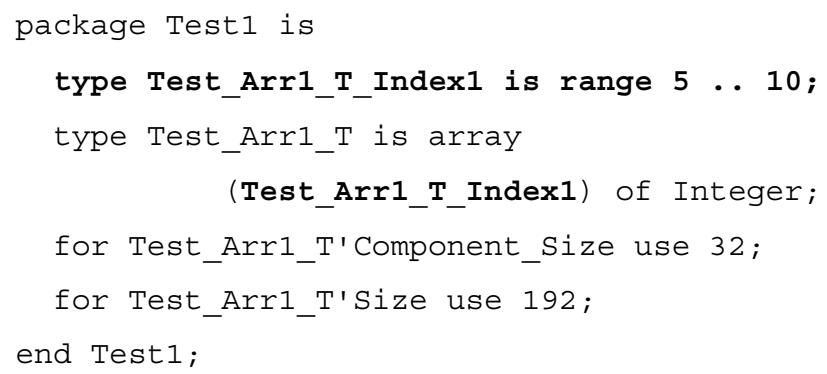

The following is the contents of the $\mathrm{C}$ header file produced:

typedef INTEGER TEST_ARR1_T[6];

As with the enumeration type above, neither the generated Ada nor C code contains the type Int_T.

\subsubsection{Anonymous Types as Fields in Records}

Ada allows an unconstrained array. Ada allows the bounds on these arrays used as a field in a record to be specified when record is declared. The tool that builds dictionary entries only supports strings as fields within records this way. In the generated type, the strings are not anonymous. For example:

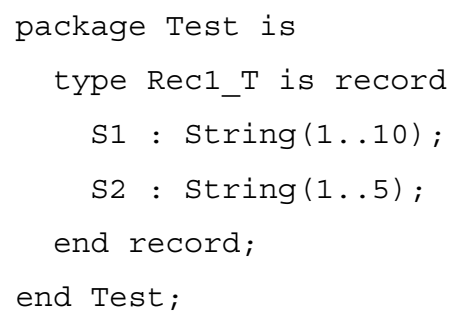

The type generator produces the following Ada spec:

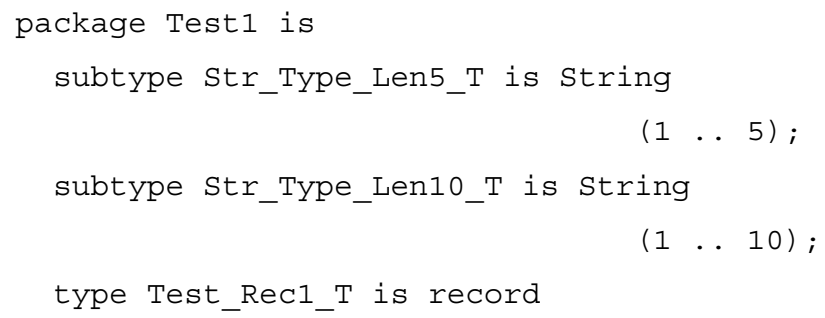

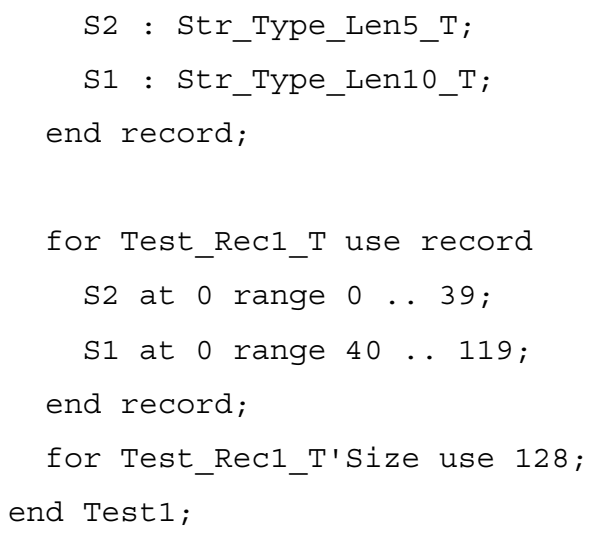

The type generator produces the following $\mathrm{C}$ header file:

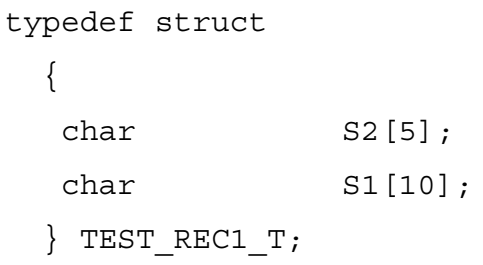

\subsection{Examples}

\subsubsection{Example of a Simple Record}

Below is a simple type which has been run through the type generator. In this example, the type is a record which contains a string and an enumeration.

Original type: Note that the fields in the record are declared in a different package (Rel) from the type itself (Rel_Msg). The type to be generated is Del_Rel_T. It does not have a rep-spec.

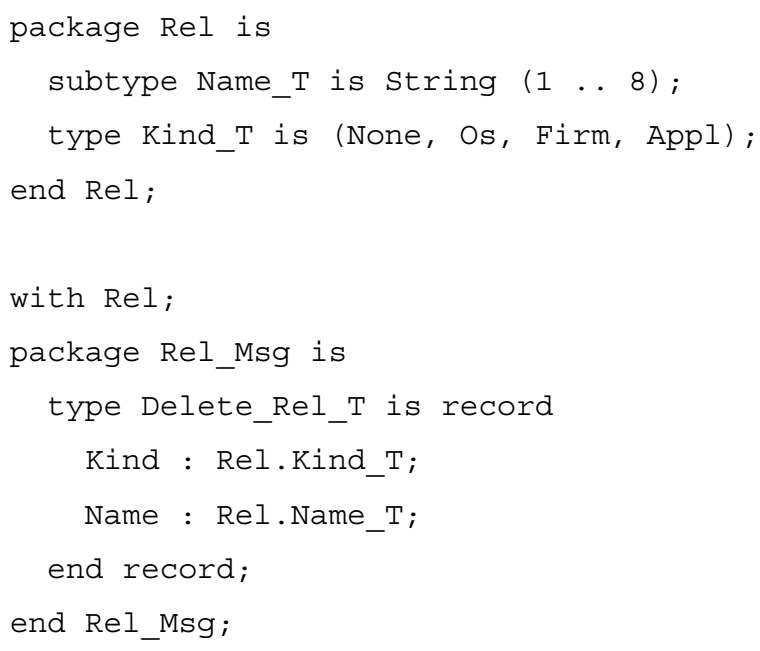

Generated Ada source code: All types the type being generated are declared in one package. Any '?' found in type names have been converted to ' '.' For example, the enumeration type in the original structure is Rel.Kind T. In the newly created Ada, the type name is Rel_Kind_T. This was done for simplicity of the type generator tool (only generates a single Ada file in a single 
package spec). Also, the newly created type has a rep-spec for the field locations and the type size to match the original type. If the generated type contained arrays, they would also have a rep-spec for the array's element size.

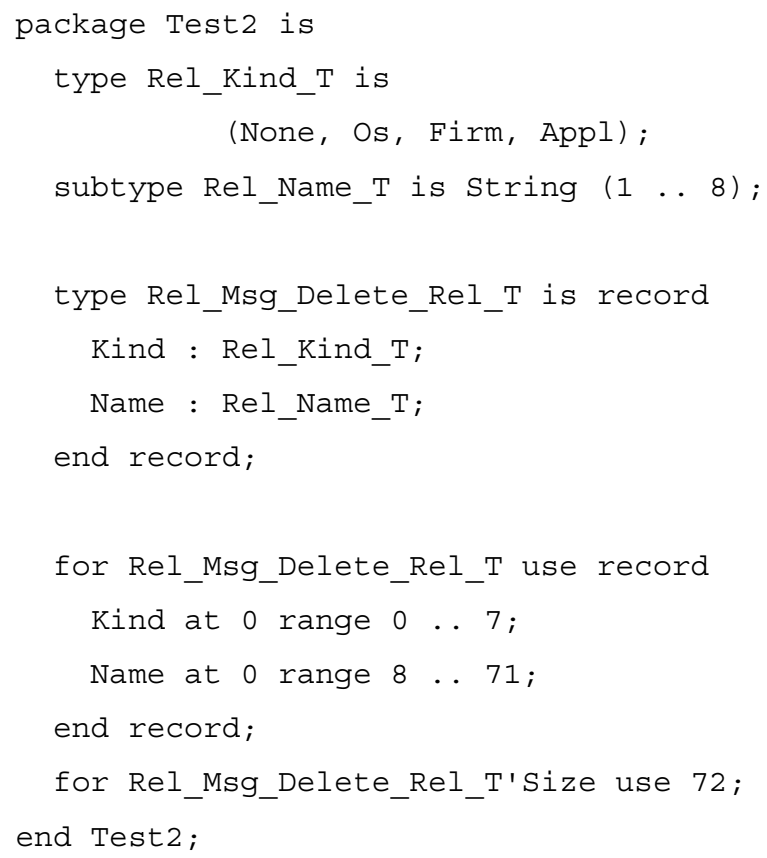

Generated C source code: As with the Ada, all types referenced are contained in a single header file. The new $\mathrm{C}$ type would have record fields ordered to match the bit layout of the original type. Also, pad fields would be inserted if necessary.

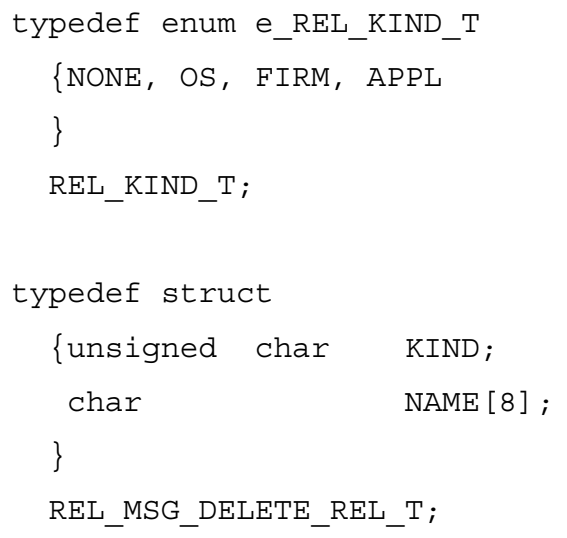

\subsubsection{More Complicated Example}

The next example is a bit more complicated, containing a record with more fields, an array of records, and a rep-spec that results in unused space. The generated $\mathrm{C}$ code will contain pad fields to fill this gap.

\section{Original type:}

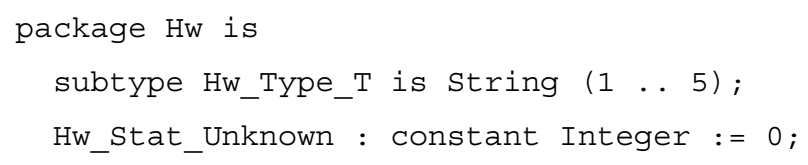

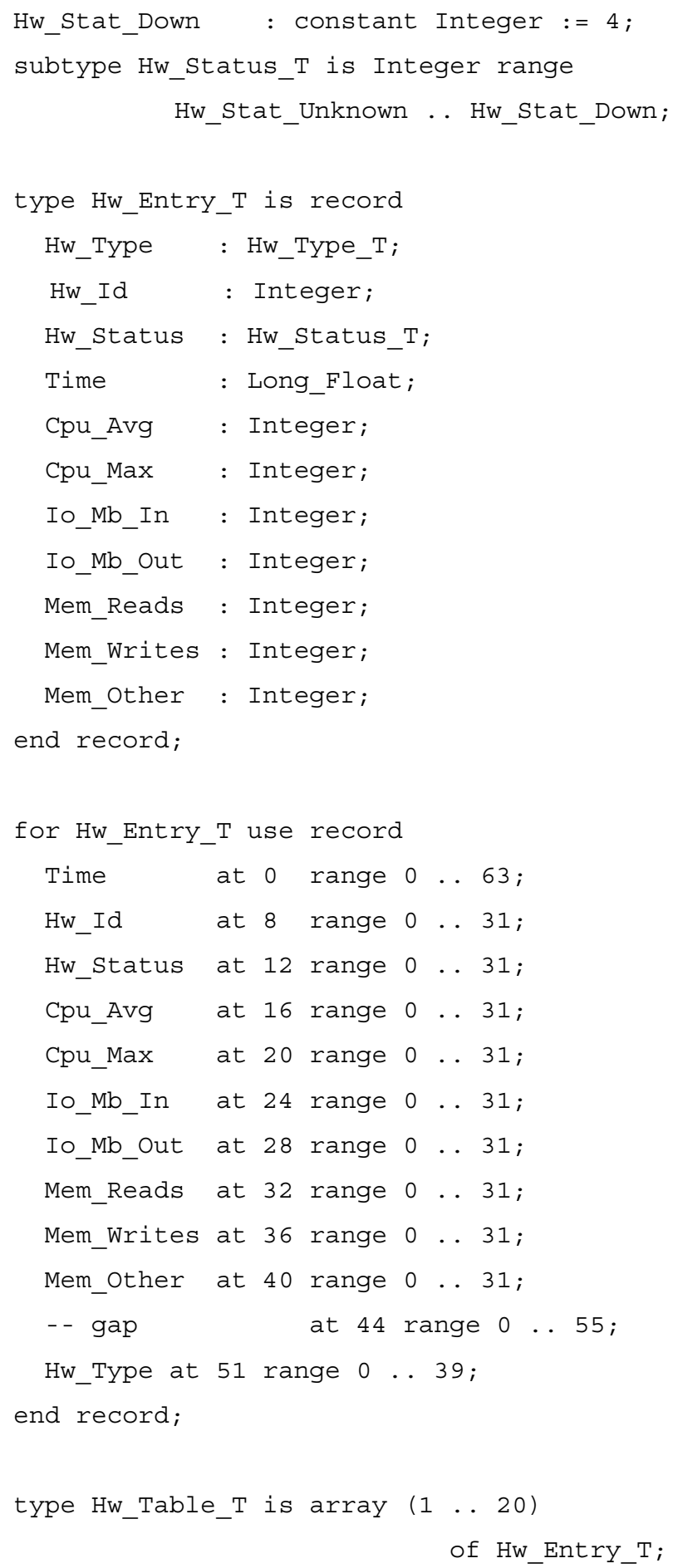

Generated Ada source code: Due to naming convention, the names in this type end up being odd (Hw_Hw_Table_T).

package Test 3 is 20

type Hw_Hw_Table_T_Index 1 is range $1 \ldots$ subtype Hw_Hw_Type_T is String (1 . . 5); 


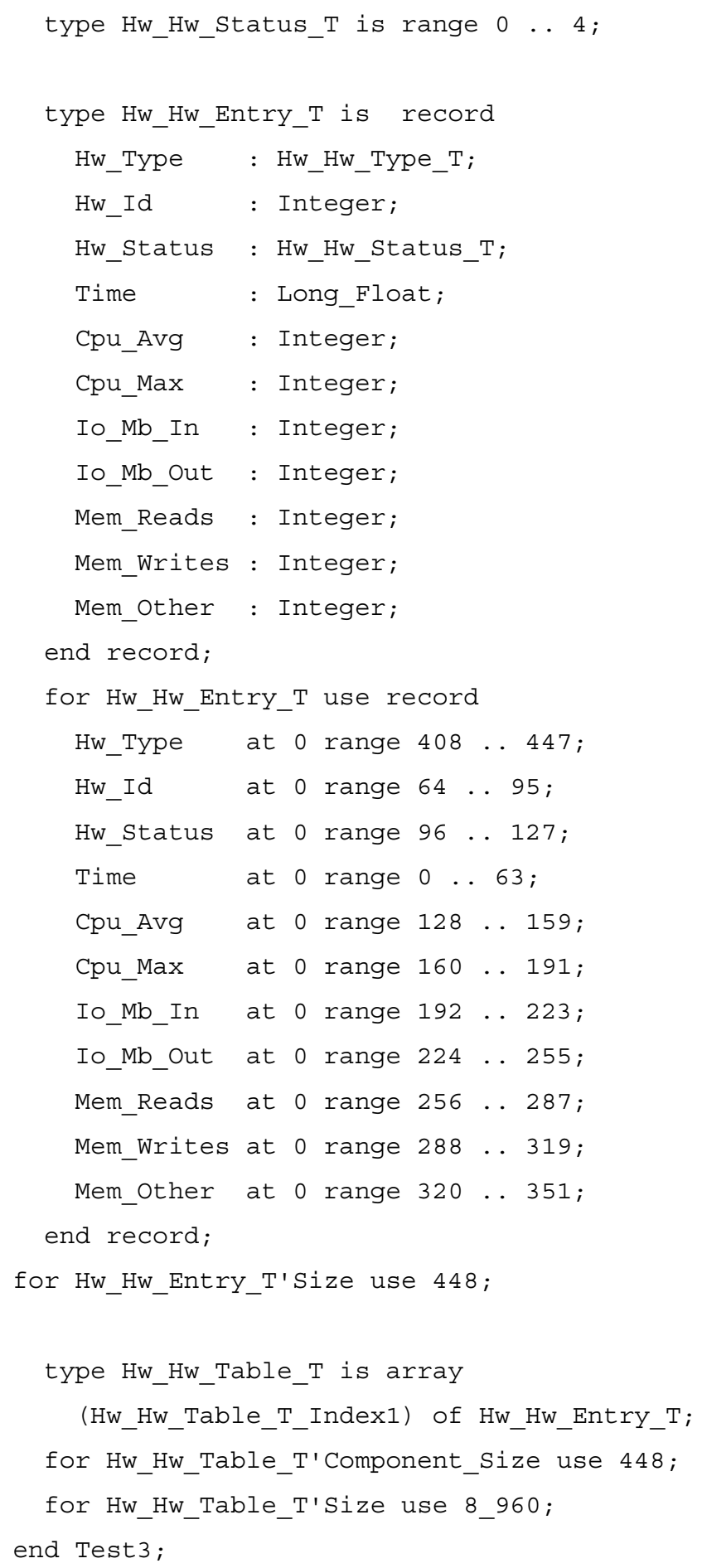

Generated C source code: Pad fields contain the following as part of the field's name "Typegen_Added_Pad_Field".

$\begin{array}{lll}\text { typedef struct }\{ & \\ \text { double } & & \text { TIME; } \\ \text { signed int } & \text { HW_ID; } \\ \text { unsigned int } & \text { HW_STATUS; }\end{array}$

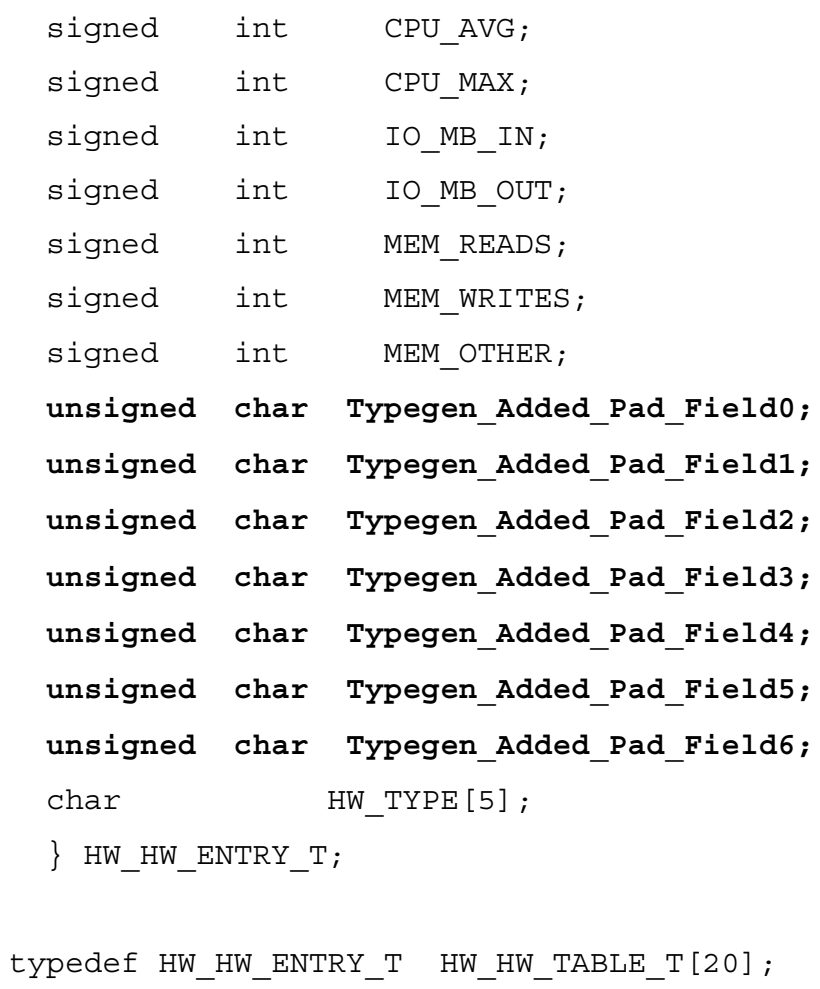

\subsubsection{Original Type Defined in $C$}

In the following example, the original type is defined in $\mathrm{C}$. Again, the generated $\mathrm{C}$ code includes pad fields; compiler alignment is not assumed.

\begin{tabular}{|c|c|c|}
\hline & \#include "/usr/include/time.h" \\
\hline typedef uns & igned cho & ar Acid[8]; \\
\hline typedef uns & igned chà & ar Tail_no[10]; \\
\hline \multicolumn{3}{|c|}{ typedef struct } \\
\hline \{unsigned & char & version; \\
\hline signed & int & peno; \\
\hline signed & short & orig; \\
\hline signed & short & dest; \\
\hline signed & int & suborig; \\
\hline signed & int & subdest; \\
\hline signed & short & sysorig; \\
\hline struct & timespec & trtm; \\
\hline struct & timespec & tmstp; \\
\hline Acid & & acid; \\
\hline Tail_no & & Tail; \\
\hline$\}$ & & \\
\hline msghd; & & \\
\hline
\end{tabular}


Generated Ada source code: Character arrays in C type are created as arrays in Ada, not strings. This is an example of why we decided to allow the generated code to be modified. Modification allows for more natural definitions in each language. If the character array were changed to a string in the Ada type, the type matching program treats an array of characters as a compatible with a string of the same size.

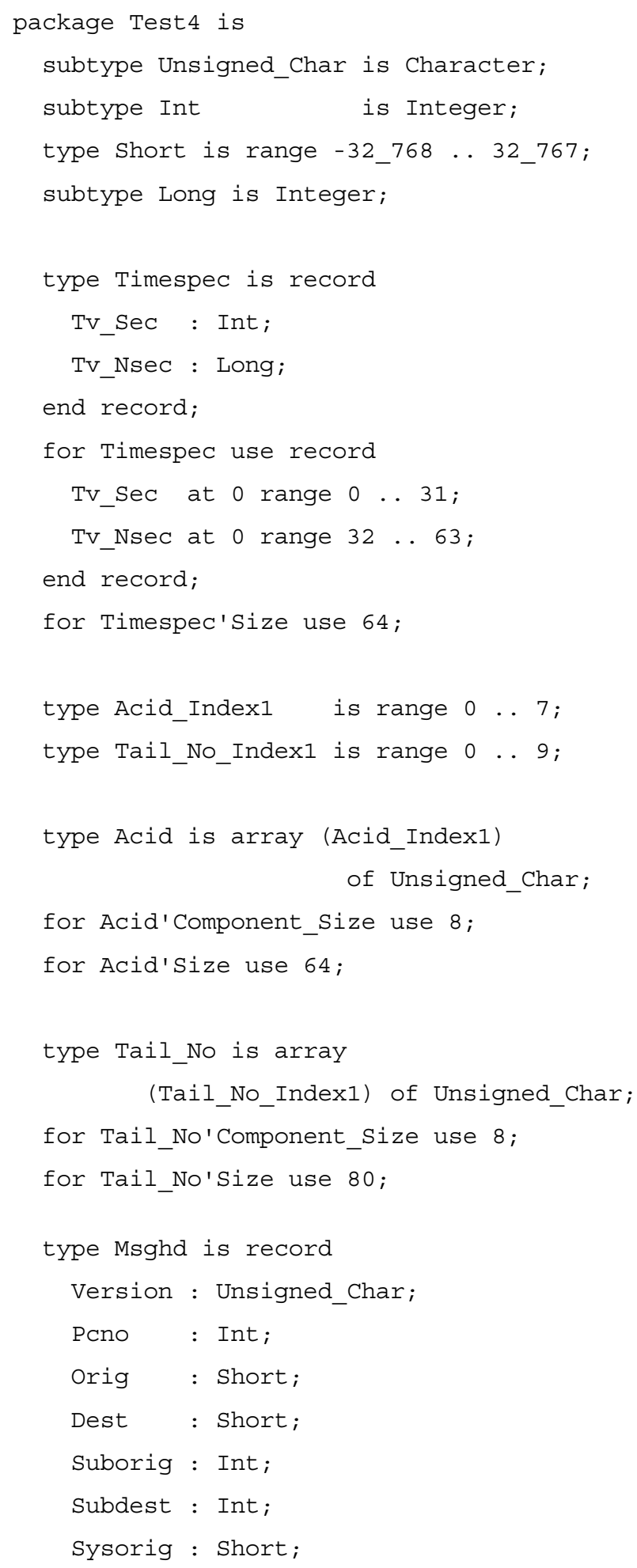

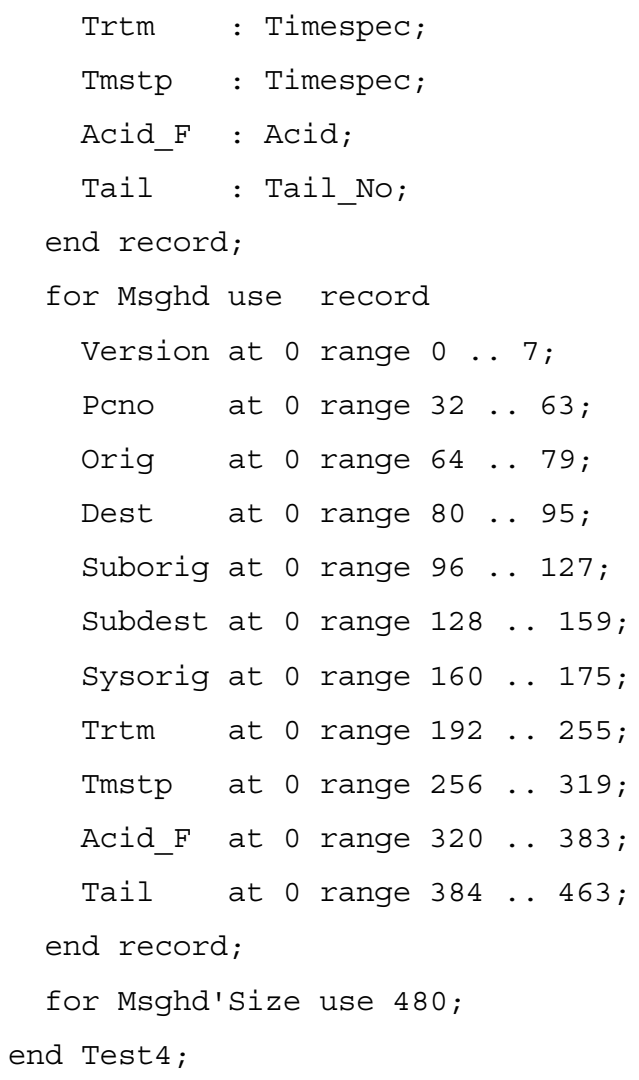

Generated C source code: Type referenced from /usr/include/time.h is included in this header file.

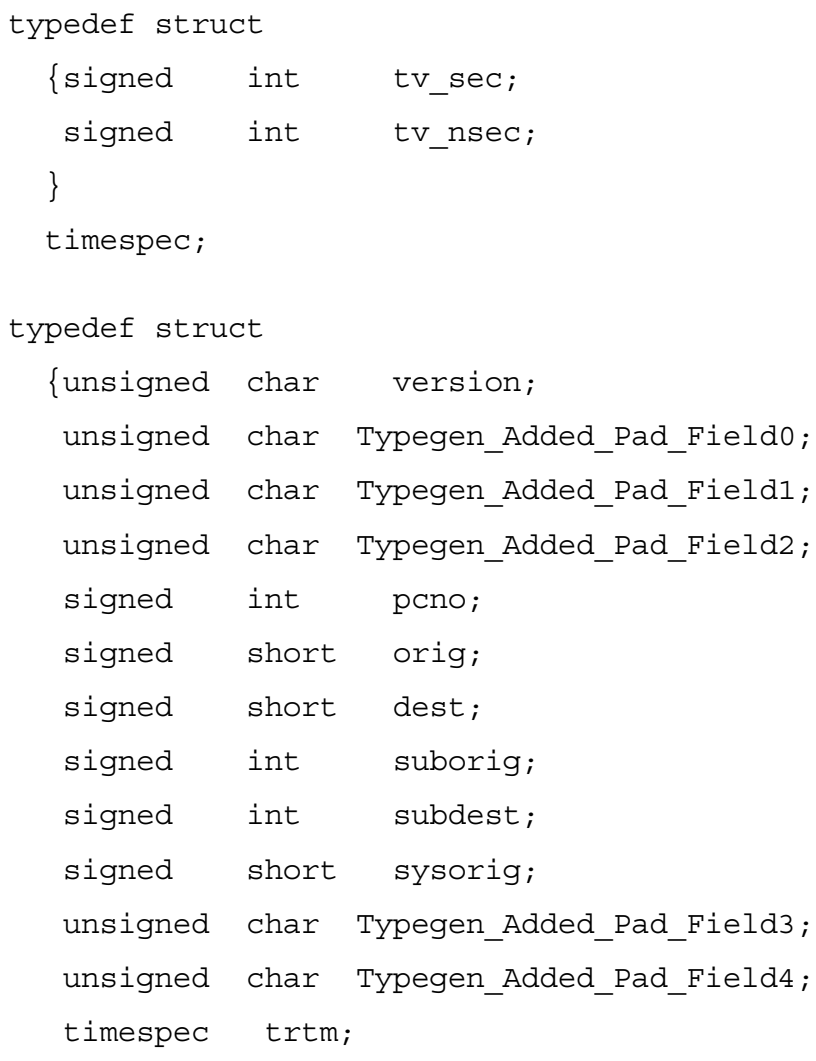




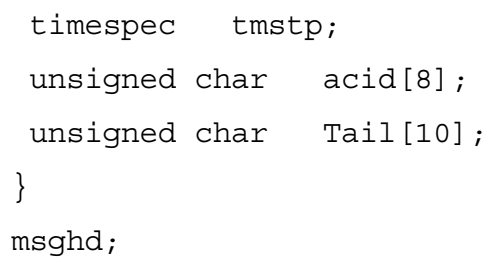

\section{TYPE MATCHING}

As mentioned earlier, the type generator program written is an aide for application developers that want to define types in Ada and $\mathrm{C} / \mathrm{C}++$ that are binary compatible. We decided to allow generated types to be modified by developers. An option could have been to have a scheme where types that were to match another in a different language were automatically generated at system build time. This presented complications. As has been shown, the naming convention of fields and types is not as the developer would have originally written. Also, generated types as currently produced are completely defined in a single package or header file and that can lead to duplication of lower level types.

Since we wanted to allow types to be modified by the owning developer, we wanted to automate checks that the types in the different languages that should match one another did.

The developer that maintains $\mathrm{Ada}$ and $\mathrm{C} / \mathrm{C}++$ types that need to be binary compatible must tool these types into dictionary entries (there are tools in place to do this), putting them in a language independent format. The type matching tool will iterate through the two types ensuring they are binary compatible.

To be binary compatible, the types being compared and all their subcomponents must meet the following conditions. The term "bit location" below refers to the relative bit offsets at which a field within a structure or array is allocated in memory. This includes start byte, start bit, end byte, and end bit.

- For array types and fields, the bit locations, the number of elements, and the element sizes, must match.

- $\quad$ For record types and fields, the bit locations must match.

- $\quad$ For integer fields, the bit locations must match and the types must have overlapping ranges.

- For floating point fields, the bit locations must match and the types must have overlapping ranges.

- $\quad$ For string fields, the bit locations must match or must match the bit location of a character array.

- For enumeration fields, the bit locations must match. An enumeration field in one type matches an integer field in the other type, provided that the bit locations of these fields match. If the fields in both types are enumerations, the number of enumeration values must match. Furthermore, the names of corresponding enumeration literals must either be the same or one must be a sub-string of the other.

- Field names containing "_pad_" will be ignored. This check is not case sensitive. These fields are assumed to be pad fields (for example, fields in a $\mathrm{C}$ structure used to consume space in order to match an Ada type whose rep-spec indicates that there are gaps between fields).
- Field names do not need to match. However, the type matching program can optionally check for field names to match.

At system build time, the type matching tool is run against all pairs of types developers have indicated should match one another. Reports are generated when these types do not match and changes are made before the system is handed off to the integration team.

\section{OTHER APPLICATIONS}

The initial focus of writing type generator and type matching programs was cross-language compatibility. Other possible applications for such a scheme are:

- Systems that transition to new compilers or platforms and go through periods where to old and new co-exist. Data structures passed between "new" and "old" software can be tooled, and type generator can then create binary compatible types. Since it creates types in Ada and $\mathrm{C} / \mathrm{C}++$, this scheme can be used to ensure compatibility of two types in the same language built with different compilers or on different platforms.

- Sharing data structures between support and operational software. We have support software data analysis programs that take recorded data structures and characterize system behavior. These programs have traditionally been written independent of the operational software. Interpretation of recorded data structures has been done forcing the data structure to be defined in a common area (the disadvantage of this approach is that the data structures may not be encapsulated as they would have been otherwise) or making queries of dictionary entries (the disadvantage of this approach is that resulting source code is more cumbersome to write than if the type was available to code against). Using the type generator, each area can have their own definition of the same type and build time checks ensure they match one another.

\section{CONCLUSION}

It is not only possible, but has also been useful to create Ada and C compatible types from dictionaries (containing data structures in a language-independent format). Maintenance effort is reduced with the help of automation. Performance is enhanced by the exchange of binary structures (i.e., by avoiding conversion of the structures to and from a language-independent format, such as XML). Compatibility between $\mathrm{Ada}$ and corresponding $\mathrm{C} / \mathrm{C}++$ data structures is verified at system build time to catch errors before handoff to test organizations.

\section{ACKNOWLEDGMENTS}

This work was performed under contract from the Federal Aviation Administration, DTFA01-03-C-00015 with the support and review from Jeff O'Leary, ERAM Product Team Software Lead, Federal Aviation Administration.

\section{REFERENCE}

[1] Mike Glasgow, Donna Hepner, and Richard Schmidt, Implementing a Table-Driven Types Dictionary Service in Ada, 1992 